\title{
Density distributions of superheavy nuclei
}

\author{
J. C. Pei, ${ }^{1}$ F. R. Xu, ${ }^{1,2,3, *}$ and P. D. Stevenson ${ }^{4}$ \\ ${ }^{1}$ School of Physics, Peking University, Beijing 100871, China \\ ${ }^{2}$ Institute of Theoretical Physics, Chinese Academy of Sciences, Beijing 100080, China \\ ${ }^{3}$ Center of Theoretical Nuclear Physics, National Laboratory of Heavy Ion Collisions, Lanzhou 730000, China \\ ${ }^{4}$ Department of Physics, University of Surrey, Guildford GU2 7XH, United Kingdom
}

(Received 8 November 2004; published 7 March 2005)

\begin{abstract}
We employed the Skyrme-Hartree-Fock model to investigate the density distributions and their dependence on nuclear shapes and isospins in the superheavy mass region. Different Skyrme forces were used for the calculations with a special comparison to the experimental data in ${ }^{208} \mathrm{~Pb}$. The ground-state deformations, nuclear radii, neutron-skin thicknesses and $\alpha$-decay energies were also calculated. Density distributions were discussed with the calculations of single-particle wave functions and shell fillings. Calculations show that deformations have considerable effects on the density distributions, with a detailed discussion on the ${ }^{292} 120$ nucleus. Earlier predictions of remarkably low central density are not supported when deformation is allowed for.
\end{abstract}

DOI: 10.1103/PhysRevC.71.034302

PACS number(s): 21.60.Jz, 21.10.Ft, 21.10.Gv, 27.90.+b

\section{INTRODUCTION}

Proton and neutron density distributions, the associated root-mean-square (rms) radii $R_{p}$ and $R_{n}$, and the neutron-skin thickness $\Delta R=R_{n}-R_{p}$ provide fundamental information on nuclear structure. For example, halo nuclei are charactered with long tails in density distributions. The density is a direct probe of the size of an atomic nucleus and plays an important role in the cross sections of nuclear reactions. Although charge densities can be measured from the elastic scattering of electrons, neutron densities are largely unknown. The parity-violating electron scattering has been suggested to measure neutron densities [1]. With the experimental method, the neutron densities of more nuclei can be expected to be measured. Theoretically, the calculated neutron-skin thickness can be model dependent [2].

Recently, Horowitz et al. [3] studied the relationship between the neutron skin of the spherical double magic nucleus ${ }^{208} \mathrm{~Pb}$ and the properties of neutron-star crusts, showing the importance of the knowledge of nucleon densities in understanding the equation of state of neutron-rich matter and therefore the properties of neutron stars. The heavy nucleus ${ }^{208} \mathrm{~Pb}$ has been measured to have a neutron-skin thickness of about $0.15 \mathrm{fm}$ [4]. With increasing mass number, the neutron excess becomes larger in general and it is natural to think that superheavy nuclei provide the largest neutron excesses. The heaviest nuclei also have large proton numbers and thus large Coulomb repulsive forces that push the protons to larger radii and therefore change density distributions. Novel density distributions were predicted in extraordinary $A>400$ nuclei that have bubbles and showed coupling effects between density distributions and shell structures [5-7]. The nucleon density in a bubble is reduced to be zero. Semibubble nuclei were also suggested with the considerable reduction of central densities for the $Z \geqslant 120$ nuclei [6] located around the center of the predicted island of stability of superheavy nuclei. Bender et al.

*Electronic address: frxu(c)pku.edu.cn. have investigated the density distributions of superheavy nuclei with the restriction of spherical shapes [8]. Recent progress in experiments are motivating the structure study of superheavy nuclei $[9,10]$. Many theoretical works have investigated the properties of superheavy nuclei [11-20], such as shell structure, $\alpha$ decay, and spontaneous fission. Experiments have also provided the structure information of superheavy nuclei by the in-beam study of spectroscopy [21-24]. In the present work, we investigate the density distributions of superheavy nuclei and related structure properties, with deformation effects taken into account.

\section{CALCUlations}

The deformed Skyrme-Hartree-Fock model (SHF) [25] was used in the present investigation. Pairing correlations are treated in the BCS scheme using a $\delta$-pairing force, $V_{\text {pair }}=$ $-V_{q} \delta\left(\vec{r}_{1}-\vec{r}_{2}\right)[26,27]$. The pairing strength $V_{q}(q=p, n$ for the protons and neutrons, respectively) has been parametrized throughout the chart of nuclei [27], but the actual values are dependent on Skyrme forces chosen. The detailed values of the pairing strengths can be found in Ref. [8].

Calculations are performed in coordinate space with axially symmetric shape. The density distribution of protons or neutrons is given in the two-dimensional form as follows:

$$
\rho(z, r)=\sum_{k} 2 v_{k}^{2}\left(\left|\psi_{k}^{+}(z, r)\right|^{2}+\left|\psi_{k}^{-}(z, r)\right|^{2}\right),
$$

where, $\psi_{k}^{+}$and $\psi_{k}^{-}$are the components of the wave functions with intrinsic spin $s_{z}=+\frac{\hbar}{2}$ and $-\frac{\hbar}{2}$, respectively, and $v_{k}^{2}$ is the pairing occupation probability of the $k$ th orbit. The ground states of most superheavy nuclei are expected to have axially symmetric or spherical shape [13]. In this article, we consider the most important axially symmetric deformations, $\beta_{2}$ and $\beta_{4}$.

In the present work, we investigated the densities and related structure problems of even-even superheavy nuclei with $Z=104-120$. In the SHF calculations, results are in 
TABLE I. The SkI4 calculations for the experimentally synthesized even-even superheavy nuclei. The calculated $\alpha$-decay energies $\left(Q_{\alpha}^{\text {cal. }}\right)$ are compared with experimental values $\left(Q_{\alpha}^{\text {expt. }}\right)$. The experimental data are taken from Ref. [32]. The calculation for the predicted magic nucleus ${ }^{298} 114_{184}$ is also listed.

\begin{tabular}{lcccccrr}
\hline \hline Nuclei & $\beta_{2}$ & $\beta_{4}$ & $\begin{array}{c}R_{n} \\
(\mathrm{fm})\end{array}$ & $\begin{array}{c}R_{p} \\
(\mathrm{fm})\end{array}$ & $\begin{array}{c}\Delta R \\
(\mathrm{fm})\end{array}$ & $\begin{array}{c}Q_{\alpha}^{\text {cal. }} \\
(\mathrm{MeV})\end{array}$ & $\begin{array}{c}Q_{\alpha}^{\text {expt. }} \\
(\mathrm{MeV})\end{array}$ \\
\hline${ }^{254} \mathrm{Rf}$ & 0.29 & 0.06 & 6.05 & 5.96 & 0.09 & 9.35 & 9.38 \\
${ }^{256} \mathrm{Rf}$ & 0.29 & 0.04 & 6.07 & 5.97 & 0.10 & 9.47 & 8.96 \\
${ }^{258} \mathrm{Rf}$ & 0.28 & 0.03 & 6.09 & 5.98 & 0.11 & 9.07 & 9.25 \\
${ }^{258} \mathrm{Sg}$ & 0.28 & 0.03 & 6.08 & 5.99 & 0.09 & 10.04 & 9.70 \\
${ }^{260} \mathrm{Sg}$ & 0.28 & 0.01 & 6.10 & 6.00 & 0.10 & 9.65 & 9.93 \\
${ }^{266} \mathrm{Sg}$ & 0.26 & -0.03 & 6.15 & 6.03 & 0.12 & 8.28 & 8.88 \\
${ }^{264} \mathrm{Hs}$ & 0.27 & -0.02 & 6.12 & 6.03 & 0.09 & 10.24 & 10.59 \\
${ }^{266} \mathrm{Hs}$ & 0.26 & -0.03 & 6.14 & 6.04 & 0.10 & 9.70 & 10.34 \\
${ }^{270} \mathrm{Hs}$ & 0.25 & -0.06 & 6.18 & 6.06 & 0.12 & 8.94 & 9.30 \\
${ }^{270} 110$ & 0.25 & -0.05 & 6.16 & 6.07 & 0.09 & 11.32 & 11.20 \\
${ }^{284} 112$ & 0.15 & -0.07 & 6.25 & 6.12 & 0.13 & 9.65 & 9.30 \\
${ }^{288} 114$ & 0.13 & -0.08 & 6.27 & 6.14 & 0.13 & 10.36 & 9.97 \\
${ }^{292} 116$ & 0.06 & -0.02 & 6.27 & 6.15 & 0.12 & 10.64 & 10.71 \\
${ }^{298} 114$ & 0.0 & 0.0 & 6.33 & 6.16 & 0.16 & 7.4 & \\
\hline \hline
\end{tabular}

general parameter dependent. For example, the SkI3 force predicts ${ }^{292} 120$ for the next magic nucleus beyond ${ }^{208} \mathrm{~Pb}$, whereas SLy7 and SkI4 predict ${ }^{310} 126$ and ${ }^{298} 114$ for the magic nucleus, respectively $[8,11]$. To make comparison, we used the different sets of parameters SLy4, SLy7 [28], SkI3, and SkI4 [29]. These sets of parameters have been developed recently with good isospin properties, and we note that they have been recommended by Rikovska Stone et al. for their ability to describe realistic neutron stars and the properties of asymmetric nuclear matter [30]. In the superheavy mass region, Skyrme parameter sets can reproduce experimental binding energies within a few $\mathrm{MeV}[31]$ and $\alpha$-decay energies within a few hundred kiloelectron volts [12]. Table I lists the properties of the ground states calculated with SkI4 for experimentally known even-even superheavy nuclei and the predicted magic nucleus ${ }^{298} 114$. The calculated $\alpha$-decay energies agree with experimental data within a few hundred kiloelectron volts (the largest difference with data is $640 \mathrm{keV}$ in ${ }^{266} \mathrm{Hs}$ ). Experimental binding energies can be reproduced within $\approx 4 \mathrm{MeV}$ for the nuclei listed in Table I with the SkI4 force. The obtained neutron-skin thicknesses are smaller than the calculations by the relativistic mean field (RMF) [17]. (It was pointed out that RMF calculations overestimate neutron-skin thicknesses [33].)

\section{A. Density distributions of spherical nuclei}

To test potential parameters in the calculations of densities, we calculated the density distributions of the spherical doubly magic nucleus ${ }^{208} \mathrm{~Pb}$ using the different sets of parameters. For ${ }^{208} \mathrm{~Pb}$, the charge distribution has been measured by electron scattering [34]. Figure 1 shows the calculated density distributions with comparison with the experimental charge density. It can be seen that the proton density given by the SkI4 force is closest to the experimental measurement. The

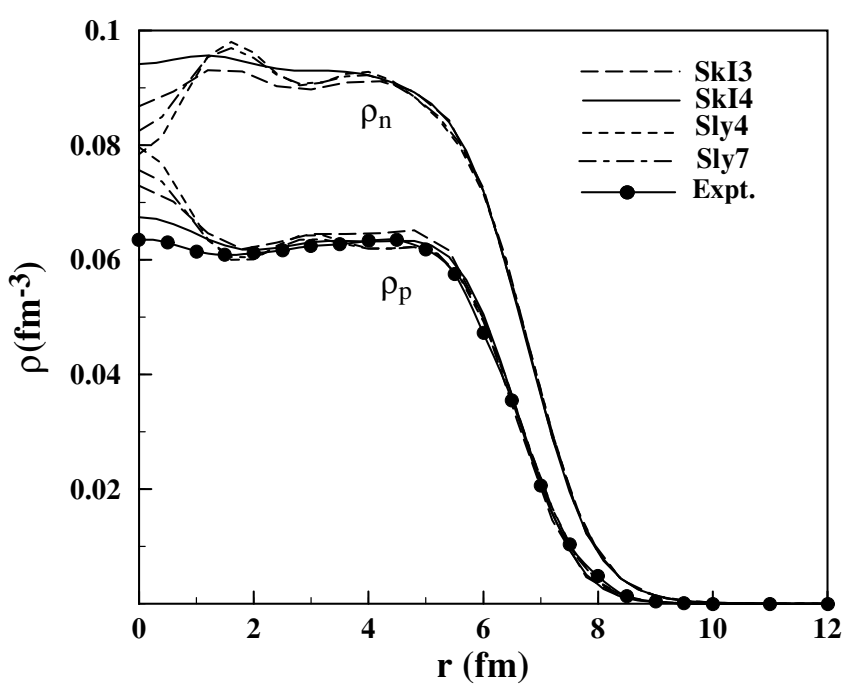

FIG. 1. The calculated proton $\left(\rho_{p}\right)$ and neutron $\left(\rho_{n}\right)$ density distributions of the spherical nucleus ${ }^{208} \mathrm{~Pb}$ compared with the experiment for the charge density [34].

rms radii of ${ }^{208} \mathrm{~Pb}$ are calculated with the SkI4 force to be $5.43 \mathrm{fm}$ for the protons and $5.61 \mathrm{fm}$ for the neutrons, leading to a neutron-skin thickness of $0.18 \mathrm{fm}$, which is slightly larger than the values of $0.16 \mathrm{fm}$ given by the SLy 4 and SLy7 forces. These results agree with the $0.15 \pm 0.02 \mathrm{fm}$ from the recent antiprotonic atom experiment [4]. The SkI3 force gives a larger neutron-skin thickness of $0.23 \mathrm{fm}$ compared to the other three forces, which may be because of the similar behavior of SkI3 force to the RMF model [8]. It needs to be pointed out that ground-state correlations (see, e.g., $[35,36]$ ) can have visible effects on nuclear properties, such as energies and densities. The oscillations observed in the calculated density distributions in Fig. 1 could be reduced when the correlation is taken into account. Such correlations, which go beyond the mean-field approximation, are not included in the present work.

Figure 2 shows the calculated densities of the spherical nucleus ${ }^{298} 114$. This nucleus was predicted to be the next doubly closed shell nucleus by a Macro-microscopic model [13] and SHF with SkI4 force [8]. For ${ }^{298} 114$, the calculations with SkI4, SLy7, and SLy4 give similar density distributions. It can be seen that the charge density distribution of ${ }^{298} 114$ has a central depression. The central density depression has been predicted to exist widely in the spherical superheavy nuclei [8]. Figure 3 displays the calculated square wave functions of the proton $1 i_{13 / 2}, 1 h_{9 / 2}$, and $2 f_{7 / 2}$ orbits that locate the $82<Z \leqslant 114$ closed shell. It can be seen that the high- $j$ orbits have density contributions in the nuclear surface region. This is consistent with the classical picture in which orbits with large angular momentum locate at the surface. In Fig. 4, we show the proton densities of ${ }^{208} \mathrm{~Pb}$ and ${ }^{298} 114$ for comparison. The proton density of ${ }^{298} 114$ is decomposed into two parts: (i) the contribution from proton orbits below the $Z=82$ closed shell and (ii) from the orbits in the next closed shell with $82<$ $Z \leqslant 114$. The contribution from the orbits below $Z=82$ has a similar behavior to the charge distribution of ${ }^{208} \mathrm{~Pb}$, without 


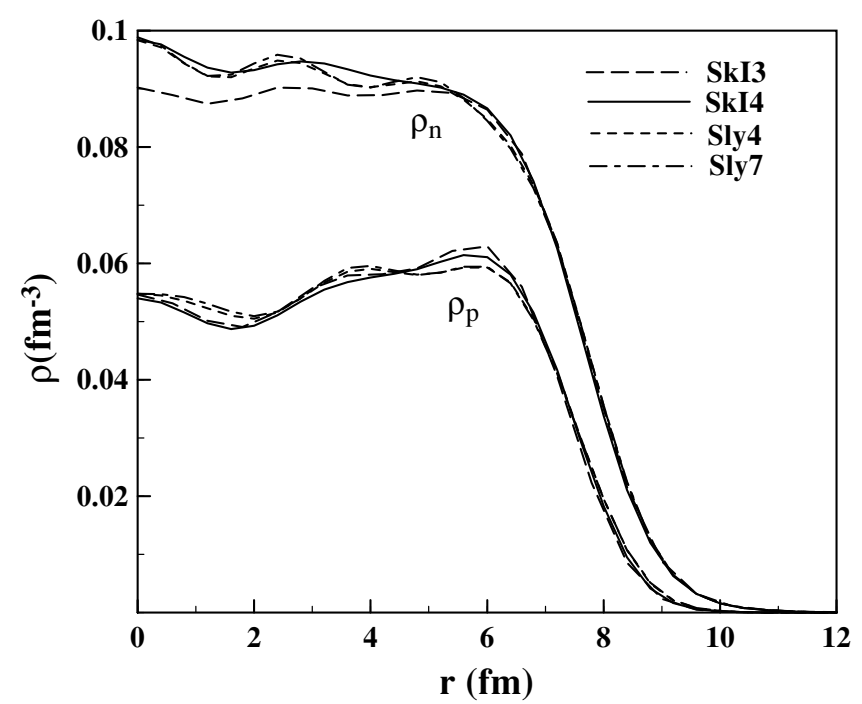

FIG. 2. The calculated proton and neutron densities for spherical nucleus ${ }^{298} 114$.

a central depression. The $82<Z \leqslant 114$ orbits (that have high $-j$ values) have contributions in the surface region of the nucleus, leading to a central depression in the proton density of ${ }^{298} 114$.

For the neutrons at spherical case, the high- $j$ orbits of $2 g_{9 / 2}$, $1 i_{11 / 2}, 1 j_{15 / 2}$, and $2 g_{7 / 2}$ appear in the region of $N=126-$ 172. The low- $j$ orbits of $4 s_{1 / 2}$ and $3 d_{3 / 2}$ are at $N=178-184$. Figure 5 shows the density distributions for $N=172-196$ and $Z=114$ with assumed spherical shape. Indeed, these nuclei were predicted to be nearly spherical in their ground states [13]. It can be seen that proton densities have central depressions and neutron densities become centrally depressed for $N \leqslant 178$.

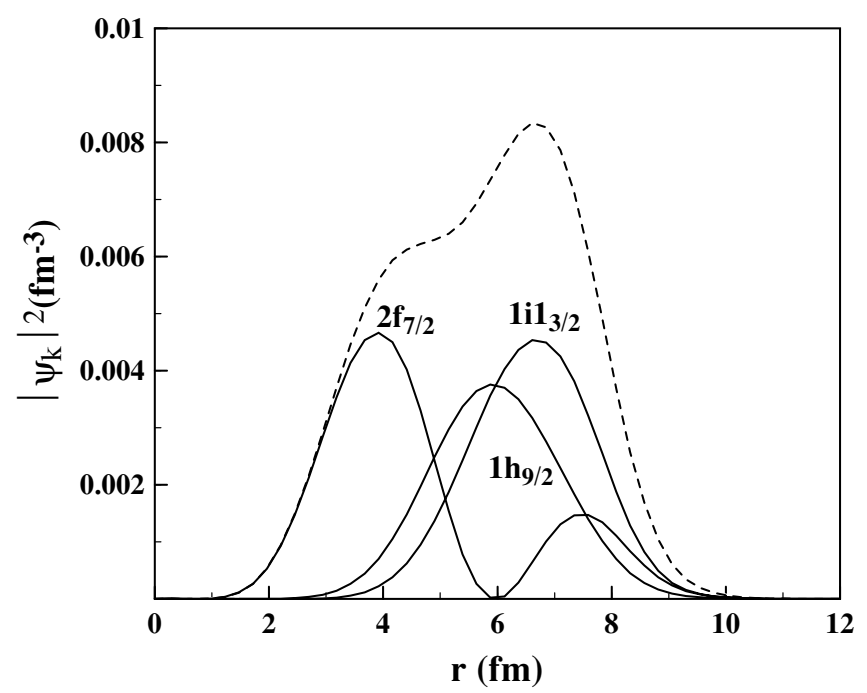

FIG. 3. The SkI4 calculated $\left|\psi_{k}\right|^{2}$ distributions of the proton $1 i_{13 / 2}, 1 h_{9 / 2}$, and $2 f_{7 / 2}$ orbits in the case of spherical ${ }^{298} 114$ nucleus. The dashed line stands for the summed contribution of the orbits.

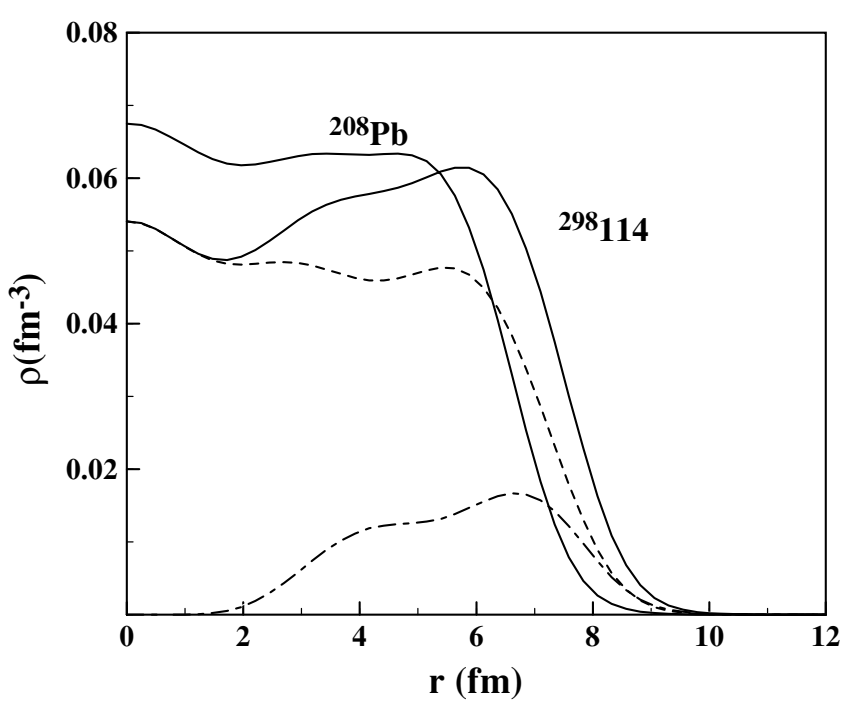

FIG. 4. The SkI4 calculated proton densities of ${ }^{298} 114$ and ${ }^{208} \mathrm{~Pb}$. In the ${ }^{298} 114$ nucleus, the dashed line presents the contribution from the orbits below the $Z=82$ closed shell, whereas the dot-dashed line presents the contribution from the high- $j$ orbits of $1 \mathrm{i}_{13 / 2}, 1 \mathrm{~h}_{9 / 2}$, and $2 \mathrm{f}_{7 / 2}$ that construct the next closed shell.

\section{B. Densities of deformed superheavy nuclei}

The shell fillings of nucleons are sensitive to the deformations of nuclei. Therefore, density distributions should be expected to be shape dependent. Most superheavy nuclei known experimentally are believed to have deformed shapes. Figure 6 displays the calculated density distributions for the $Z=110$ isotopes with $N=160,170$, and 180 nuclei. The equilibrium deformations are determined by minimizing calculated energies. The deformations determined with the SkI4 force are $\beta_{2}=0.25,0.19$, and 0.05 for ${ }^{270,280,290} 110$, respectively. To check the possible parameter dependence, we also used the SLy7 force to calculate the densities,

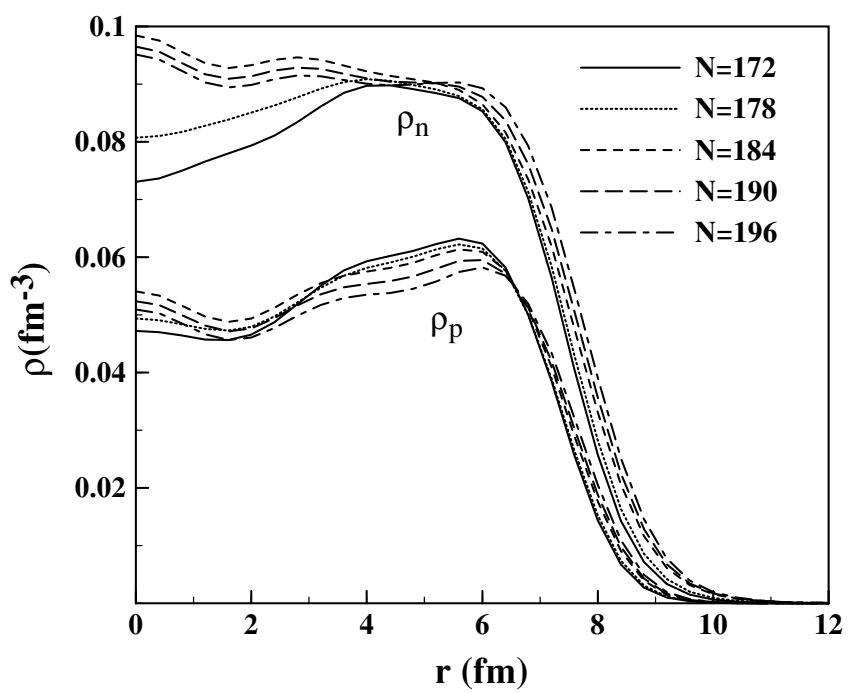

FIG. 5. The spherical density profiles of the nuclei with $Z=114$ and $N=172-196$ calculated with the SkI4 force. 


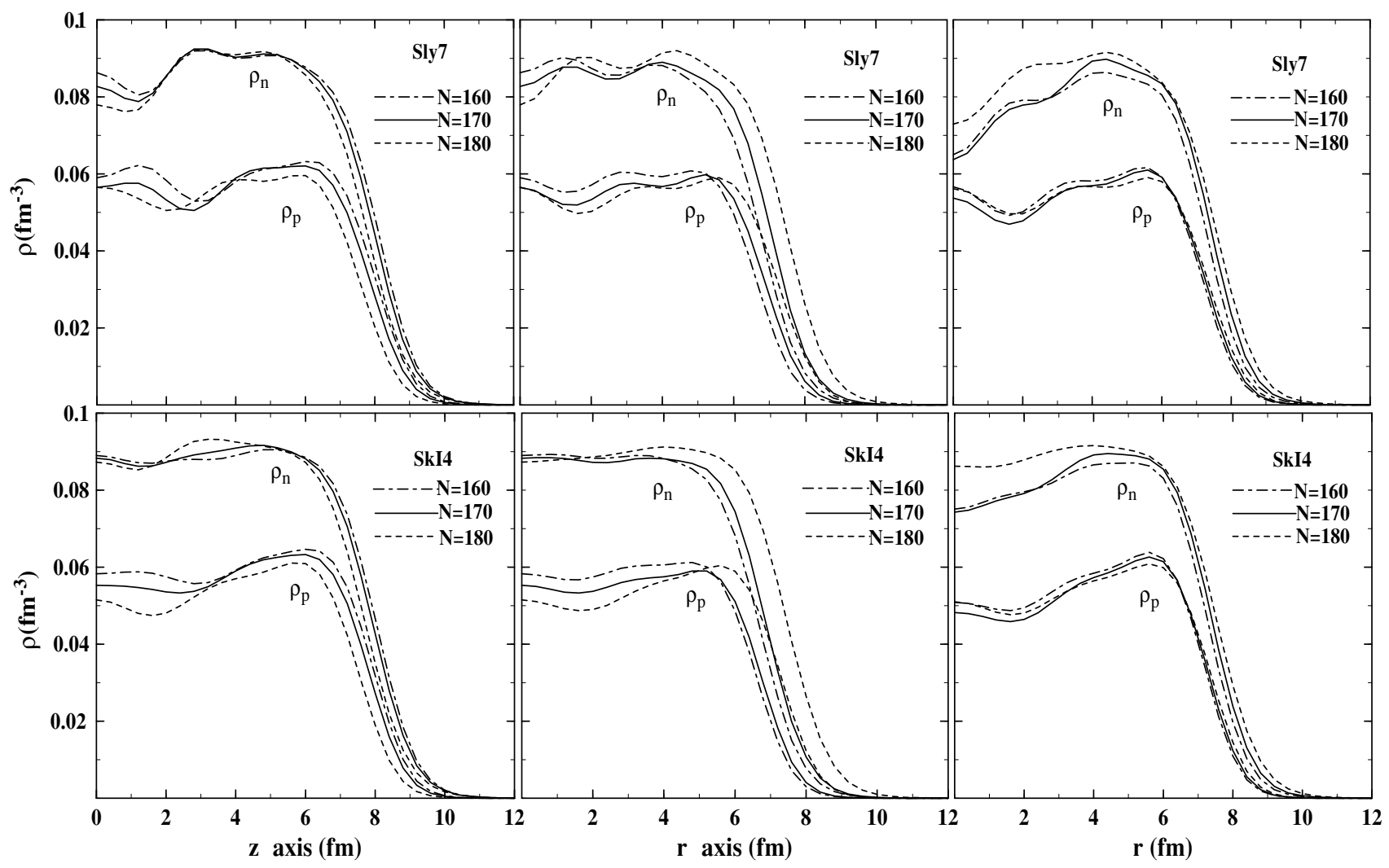

FIG. 6. Calculated density distributions of nuclei of the deformed $270,280,290110$. The left and middle columns show the distribution profiles of $\rho(z, 0)$ along the $z$ axis and $\rho(0, r)$ along the $r$ axis, respectively. For comparison, distributions at assumed spherical shape are also displayed in the right column.

shown in Fig. 6. The deformations determined with SLy7 are $\beta_{2}=0.25,0.17$, and 0.05 for ${ }^{270,280,290} 110$, respectively. To see deformation effects, we calculated the densities assuming spherical shape. It can be seen for ${ }^{270,280} 110$ that the densities become more centrally depressed in spherical cases. We also see some difference between the densities along the $z$ and $r$ axes. Figure 7 shows the two-dimensional proton density distribution for the ${ }^{280} 110$ nucleus. The density has two humps in the $z$ axis. The double-hump distribution has been suggested experimentally, for example, in the deformed ${ }^{166} \mathrm{Er}$ and ${ }^{176} \mathrm{Yb}$ [37]. Our calculations with SkI4 and SLy7 show that such a double-hump phenomenon is relatively pronounced for nuclei around ${ }^{280} 110$. The macro-microscopic calculations show that the even-even nuclei around $N=170$ are particularly unstable against spontaneous fission (see Fig. 9 in [13]). This could be related to the double-hump distributions in these deformed nuclei.

Figure 8 shows the distributions of the square wave functions of the $j_{z}=1 / 2$ orbits in ${ }^{280} 110$, modified with pairing occupation probabilities [see Eq. (1)]. These orbits are above the $Z=82$ and $N=126$ shells for the protons and neutrons, respectively. Shown as in Fig. 8, the high-j, low $-j_{z}$ orbits have important contributions to densities in the surface region of the $z$ axis. High- $j$, low- $j_{z}$ orbits have strong prolate-driving effect. Hence, sufficient number of high- $j$, low $-j_{z}$ orbits occupied can result in prolate shapes and double-hump densities. In ${ }^{166} \mathrm{Er}$ and ${ }^{176} \mathrm{Yb}$ that were suggested experimentally to have double-hump densities, the low- $j_{z}$ orbits of the proton $1 h_{11 / 2}$, $1 g_{7 / 2}$ subshells are occupied.

Density distributions given by different Skyrme forces can differ, as shown in Fig. 6. The SLy7 predicts a larger central depression in neutron densities and a less central depression in proton densities than the SkI4 force. This

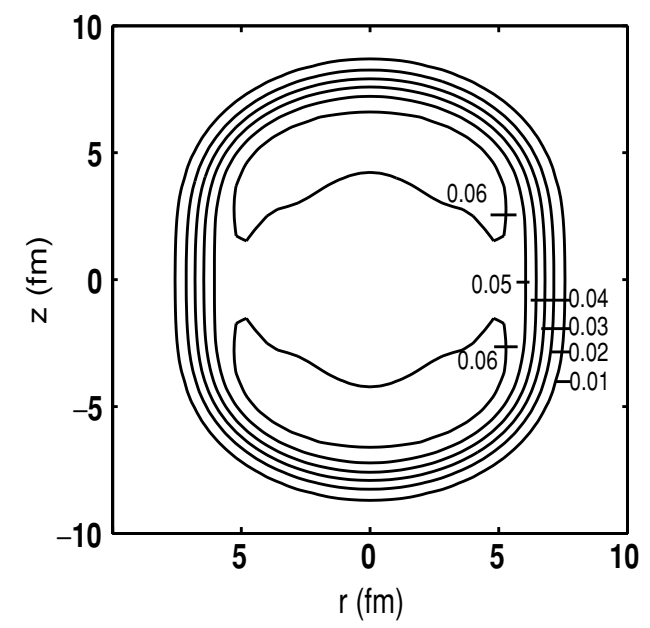

FIG. 7. Two-dimensional proton density contours of the nucleus ${ }^{280} 110$, calculated with the SkI4 force. The numbers on the contours are the values of densities in $\mathrm{fm}^{-3}$. 


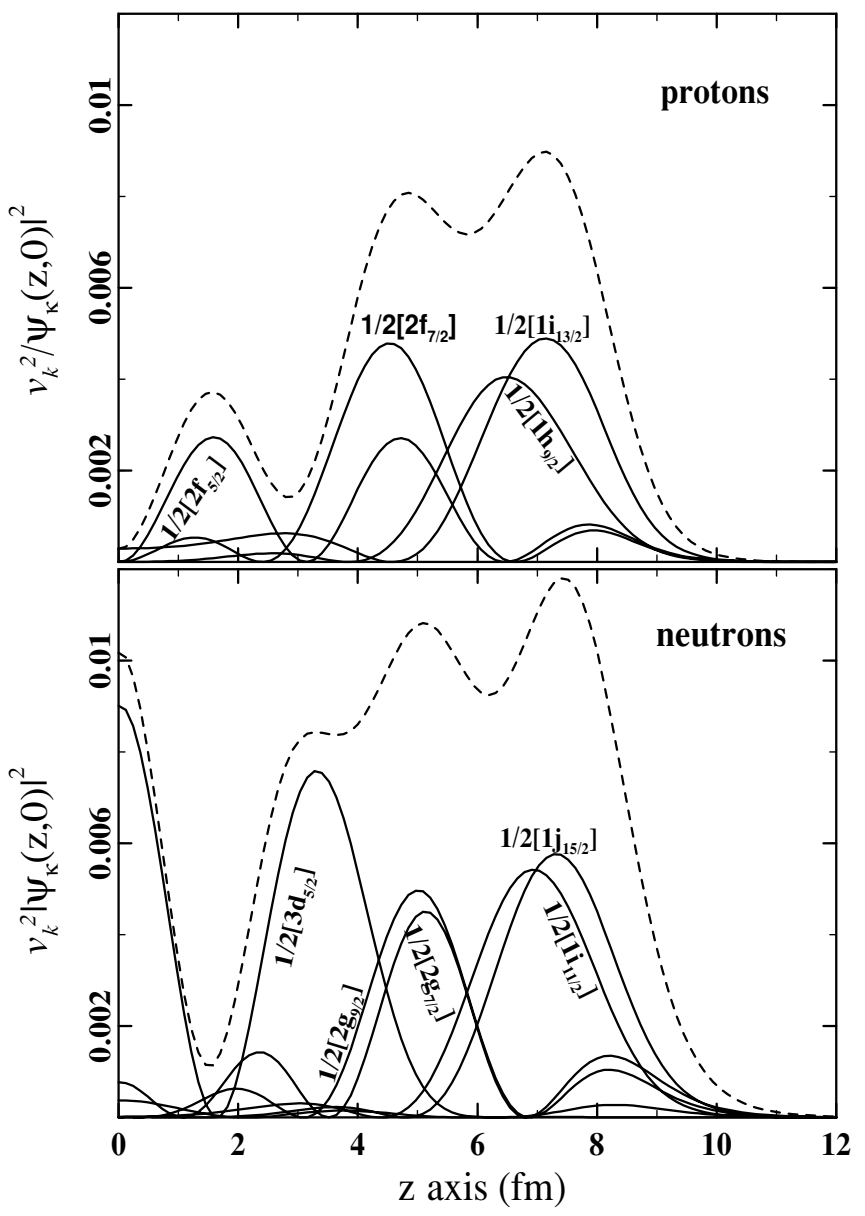

FIG. 8. The SkI4-calculated contributions (in the $z$ axis) of the $j_{z}=1 / 2$ orbits in the ${ }^{280} 110$ case, with the modifications of pairing occupation probabilities. The dashed line represents the summed contributions of the orbits.

difference also occurs in the calculation of ${ }^{208} \mathrm{~Pb}$ (see Fig. 1). For different parameters, the opposite behavior of proton and neutron distributions reduces the difference in the total (proton+neutron) nuclear density distributions. The origin of the opposite behavior would be because of the self-consistent coupling between protons and neutrons in the SHF model to approach the nuclear density saturation [38]. The SkI4 force is better in reproducing the density of ${ }^{208} \mathrm{~Pb}$ compared to other Skyrme forces used in the present investigation. However, the SkI4 force was shown to have larger spin-orbit splittings in the calculations of single-particle level schemes for the superheavy region [8].

\section{The ${ }^{292} 120$ nucleus}

${ }^{292} 120$ is an interesting nucleus that was predicted to be a doubly magic [8] and spherical semibubble nucleus [6]. We calculated the energy curve with the SLy4, SLy7, SkI3, and SkI4 parameters; see Fig. 9. The results of SLy4, SLy7, and SkI4 are close to the Hartree-Fock calculations with Gogny force [6]. The SkI3 force gives a shallow spherical minimum. The SkI4 calculation predicts three minima at $\beta_{2}=0.11$, -0.12 , and 0.52 (superdeformations in superheavy nuclei have

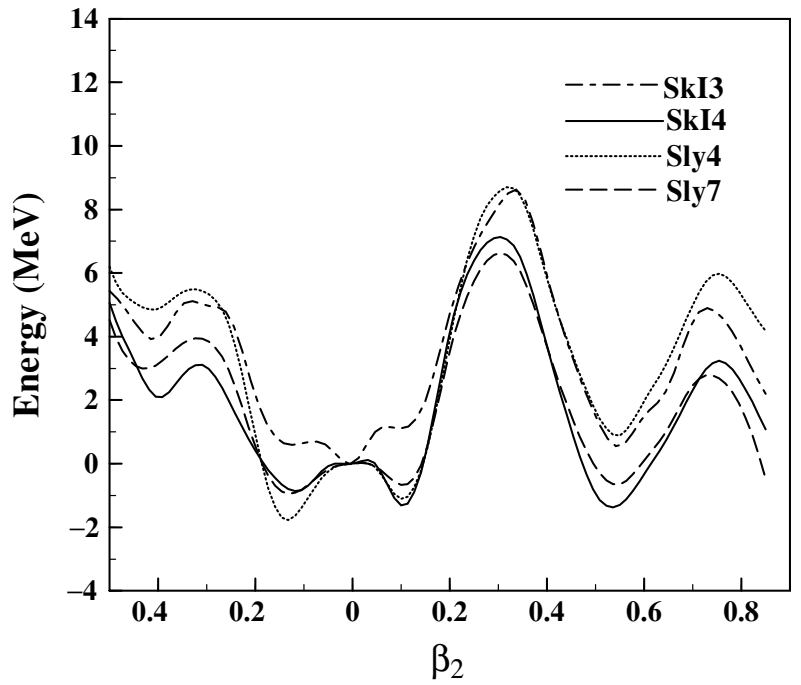

FIG. 9. Calculated energy curves of ${ }^{292} 120$ with different Skyrme forces as a function of quadrupole deformation.

been discussed by Ren et al. [16,17]). It needs to be mentioned that the present calculations are restricted to axially symmetric shapes without considering the possibility of triaxiality. The inclusion of the triaxial degree of freedom could alter the shallow minima. Figure 10 shows the SkI4 calculated density distributions at the prolate and oblate shapes. For comparison, the densities at the spherical shape are also displayed. It can be seen that significant central depressions or central semibubble appear in the spherical case. However, the situation is considerably altered even with a small shape change. Only weak central depressions are seen at the small deformations (see Fig. 10). To have a further understanding, we calculated the corresponding single-particle potentials in the $z$ axis, shown inside Fig. 10. It can be seen that the proton potential has a considerable change with changing the deformation. The spherical proton potential has a significant hump at the center of the nucleus. This implies that the Coulomb energy can be considerably reduced by forming the center semibubble at the spherical shape. The Coulomb energy can also be reduced by generating the deformation of the nucleus. In the reduction of the total energy of the nucleus, there is competition between forming the central semibubble and generating the deformation. The deformation can affect the shell structure and then density distributions, and vice versa. In a self-consistent model, such as the SHF approach, densities are fed back into the potential, which amplifies the coupling between deformations and densities.

\section{SUMMARY}

In summary, the density distributions of superheavy nuclei have been investigated with the Skyrme-Hartree-Fock model. To test the model and parameters, the $\alpha$-decay energies of even-even superheavy nuclei and the charge density of ${ }^{208} \mathrm{~Pb}$ are calculated and compared to existing experimental data. For axially symmetrically deformed nuclei, the density distributions in the symmetric $z$ and the vertical $r$ axes or the 


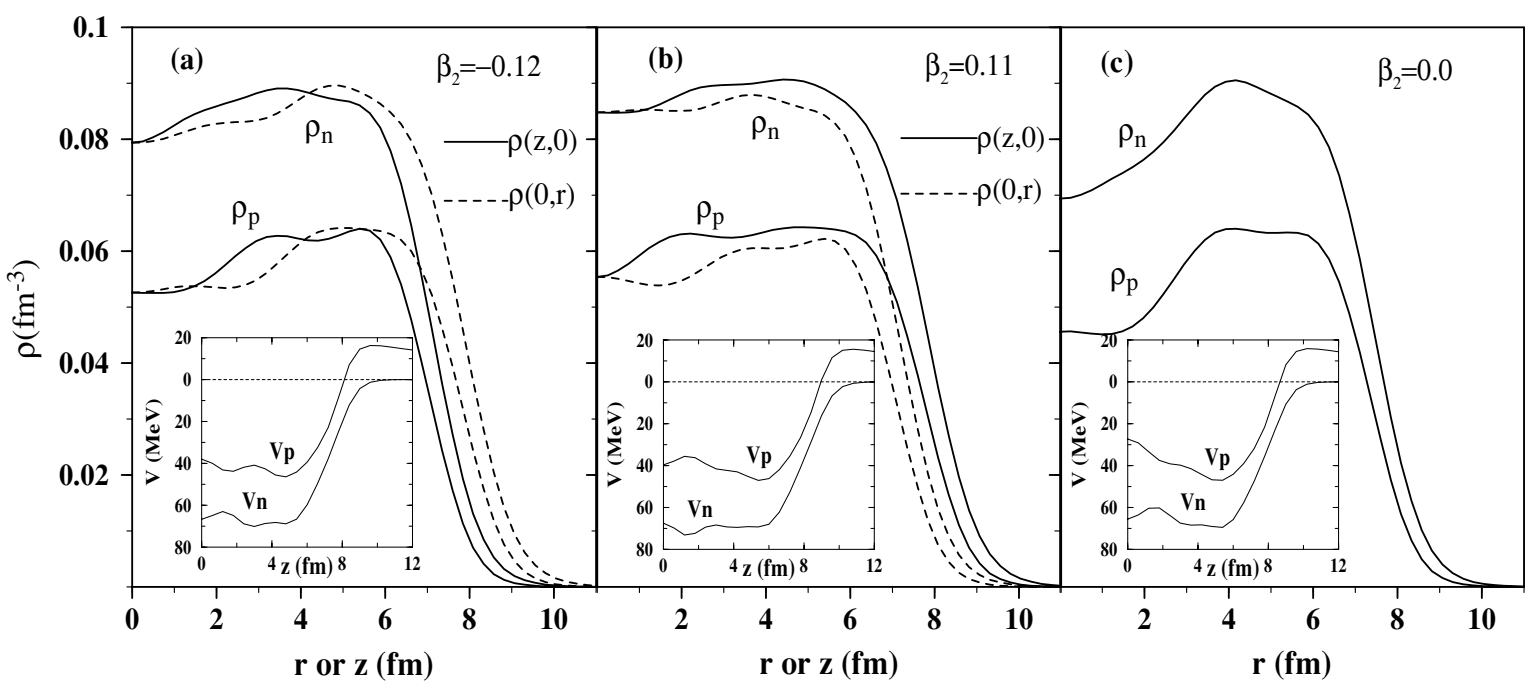

FIG. 10. The SkI4 calculated density distributions $\rho(z, 0)$ and $\rho(0, r)$ in ${ }^{292} 120$ at $\beta_{2}=-0.12$ (a), 0.11 (b), and 0.0 (c), corresponding to the energy minima shown in Fig. 9 and the spherical shape. The inset figures show the single-particle potentials in the $z$ axis.

two-dimension distributions were calculated. The distribution in different directions can be different. The high- $j$, low $-j_{z}$ orbits have important contributions to the densities at nuclear surface in the $z$ axis, whereas high- $j$, high- $j_{z}$ orbits have important contributions at surfaces in the $r$ axis. The deformation effect was found to be significant in the calculation of the density distribution in the ${ }^{292} 120$ nucleus. Only a weak central depression was seen in the deformed case for ${ }^{292} 120$ compared to the predicted semibubble at the spherical shape.

\section{ACKNOWLEDGMENTS}

We thank Professor P. M. Walker for his valuable comments and Professor P.-G. Reinhard for the computer code. This work was supported by the Chinese Major State Basic Research Development Program No. G2000077400, the Natural Science Foundation of China (Grants 10175002 and 10475002), the Doctoral Foundation of Chinese Ministry of Education (20030001088), the U.K. Royal Society, and the U.K. Science and Engineering Research Council.
[1] T. W. Donnelly, J. Dubach, and Ingo Sick, Nucl. Phys. A503, 589 (1989).

[2] C. J. Horowitz, S. J. Pollock, P. A. Souder, and R. Michaels, Phys. Rev. C 63, 025501 (2001).

[3] C. J. Horowitz and J. Piekarewicz, Phys. Rev. Lett. 86, 5647 (2001).

[4] A. Trzcińska, J. Jastrzebski, P. Lubiński, F. J. Hartmann, R. Schmidt, T.von Egidy, and B. Kłos, Phys. Rev. Lett. 87, 082501 (2001).

[5] K. Dietrich and K. Pomorski, Phys. Rev. Lett. 80, 37 (1998); Nucl. Phys. A627, 175 (1997).

[6] J. Dechargé, J.-F. Berger, K. Dietrich, and M.S. Weiss, Phys. Lett. B451, 275 (1999).

[7] Y. Yu, A. Bulgac, and P. Magierski, Phys. Rev. Lett. 84, 412 (2000).

[8] M. Bender, K. Rutz, P.-G. Reinhard, J. A. Maruhn, and W. Greiner, Phys. Rev. C 60, 034304 (1999).

[9] Y. T. Oganessian et al., Nature, 400, 242 (1999); Phys. Rev. C 63, 011301(R) (2001).

[10] S. Hofmann and G. Münzenberg, Rev. Mod. Phys. 72, 733 (2000).

[11] S. Ćwiok, J. Dobaczewski, P.-H. Heenen, P. Magierski, and W. Nazarewicz, Nucl. Phys. A611, 211 (1996).
[12] S. Ćwiok, W. Nazarewicz, and P.-H. Heenen, Phys. Rev. Lett. 83, 1108 (1999).

[13] R. Smolańczuk, Phys. Rev. C 56, 812 (1997).

[14] W. Nazarewicz, M. Bender, S. Ćwiok, P. H. Heenen, A. T. Kruppa, P.-G. Reinhard, and T. Vertse, Nucl. Phys. A701, 165 (2002).

[15] M. Bender, Phys. Rev. C 61, 031302(R) (2000).

[16] Zhongzhou Ren and Hiroshi Toki, Nucl. Phys. A689, 691 (2001).

[17] Zhongzhou Ren, Phys. Rev. C 65, 051304(R) (2002).

[18] J. Meng and N. Takigawa, Phys. Rev. C 61, 064319 (2000).

[19] F. R. Xu, E. G. Zhao, R. Wyss, and P. M. Walker, Phys. Rev. Lett. 92, 252501 (2004).

[20] Z. Y. Wu, F. R. Xu, E. G. Zhao, and C. K. Zheng, Chin. Phys. Lett. 20, 1702 (2003).

[21] P. Reiter et al., Phys. Rev. Lett. 82, 509 (1999).

[22] R.-D. Herzberg et al., Phys. Rev. C 65, 014303 (2001).

[23] P. A. Butler et al., Phys. Rev. Lett. 89, 202501 (2002).

[24] R.-D. Herzberg, J. Phys. G 30, R123 (2004).

[25] V. Blum, G. Lauritsch, J. A. Maruhn, and P.-G. Reinhard, J. Comput. Phys. 100, 364 (1992).

[26] S. J. Krieger, P. Bonche, H. Flocard, P. Quentin, and M. S. Weiss, Nucl. Phys. A517, 275 (1990). 
[27] M. Bender, K. Rutz, P.-G. Reinhard, and J. A. Maruhn, Eur. Phys. J. A 8, 59 (2000).

[28] E. Chabanat, P. Bonche, P. Hasensel, J. Meyer and R. Schaeffer, Nucl. Phys. A635, 231 (1998).

[29] P.-G. Reinhard and H. Flocard, Nucl. Phys. A584, 467 (1995).

[30] J. R. Stone, J. C. Miller, R. Koncewicz, P. D. Stevenson, and M. R. Strayer, Phys. Rev. C 68, 034324 (2003).

[31] T. Bürvenich, K. Rutz, M. Bender, P.-G. Reinhard, J. A. Maruhn, and W. Greiner, Eur. Phys. J. A 3, 139 (1998).

[32] G. Audi, A. H. Wapstra, and C. Thilbault, Nucl. Phys. A729, 337 (2003).
[33] R. J. Furnstahl, Nucl. Phys. A709, 85 (2002).

[34] H. Euteneuer, J. Friedrich, and N. Vögler, Nucl. Phys. A298, 452 (1978).

[35] M. R. Strayer, W. H. Bassichis, and A. K. Kerman, Phys. Rev. C 8, 1269 (1973).

[36] N. Dinh Dang and V. Zelevinsky, Phys. Rev. C 64, 064319 (2001).

[37] T. Cooper, W. Bertozzi, J. Heisenberg et al., Phys. Rev. C 13, 1083 (1976).

[38] W. A. Richter and B. A. Brown, Phys. Rev. C 67, 034317 (2003). 